\title{
BM-QFD: UMA ABORDAGEM PARA O EMPREENDEDORISMO TECNOLÓGICO NA CRIAÇÃO DE SPIN-OFF ACADÊMICA
}

Leonel Del Rey de Melo Filho (leoneldr@pucminas.br) - Pontifícia Universidade Católica de Minas Gerais (PUC-MG)

Raoni Barros Bagno (raonibagno@ dep.ufmg.br) - Universidade Federal de Minas Gerais (UFMG)

Marina Bastos Carvalhais Barroso (marinabastosb@gmail.com) - Universidade Federal de Minas Gerais (UFMG)

Márcia Dias Diniz Costa (mdinizcosta@gmail.com) - Universidade Federal de Minas Gerais (UFMG), Instituto Nacional de Metrologia, Qualidade e Tecnologia (Inmetro)

\section{RESUMO}

Este estudo vem propor o BM-QFD como abordagem metodológica de suporte à concepção e desenvolvimento de serviços intensivos em ciência e tecnologia. Partindo dos princípios do Design Science Research e da demanda real da Startup FabNS é concebido um serviço de Nanometrologia de Grafenos baseado no uso do TERS - tecnologia de fronteira mundial e essencial para o desenvolvimento no campo da nanotecnologia. A proposta contou com a combinação e adaptação de métodos consolidados na literatura empregados tanto no desenvolvimento de novos produtos e serviços em empresas estabelecidas, quanto na atividade empreendedora de empresas nascentes. A abordagem resultante, no entanto, é inovadora em relação à forma como tais métodos são combinados em prol das particularidades do desenvolvimento de Spin-offs acadêmicas intensivas em ciência.

Palavras-Chave: Empreendedorismo Tecnológico; Spin-offs Acadêmicas; Tecnologias Gerenciais; QFD; Business Model Canvas. 


\section{INTRODUÇÃ̃o}

O empreendedorismo tecnológico pode ser compreendido como o reconhecimento, criação e exploração de tecnologias que envolvem a geração e consolidação de novas empresas de base tecnológica (EBTs) (RATINHO; HARMS; WALSH, 2015). Nos últimos anos tem havido uma forte escalada do empreendedorismo, inserindo definitivamente o termo "startup" no vocabulário de acadêmicos e praticantes. Neste campo, contribuições como os conceitos de Startup Enxuta, produto mínimo viável, Customer Development (RIES, 2011; BLANK, 2013) e outros tornaram-se o vocabulário comum na área. Essas linhas de estudo visam prover métodos e ferramental gerencial para se lidar com novos empreendimentos de base tecnológica.

Negócios nascentes em áreas de fronteira científica e tecnológica se defrontam com altos níveis de incerteza, complexidade e imprevisibilidade (SHANE, 2004, LASMAR; FREITAS, 2020). Uma abordagem sistemática baseada em processos, métodos e ferramentas combinados visa principalmente orientar os empreendedores na diminuição das incertezas e riscos durante a geração do novo negócio (SOUZA et al., 2020).

O presente estudo tem como objetivo propor uma abordagem de métodos combinados para o empreendedorismo na criação de spin-off acadêmica intensiva em ciência e tecnologia, o BMQFD, partindo da demanda real de uma startup voltada ao desenvolvimento de um serviço especializado de Nanometrologia de Grafenos.

\section{METODOLOGIA E CONTEXTO DO DESENVOLVIMENTO DO ESTUDO}

O desenvolvimento deste estudo partiu da necessidade real da startup FabNS (Fábrica de Nanosoluções) em elaborar um plano de negócios para atendimento à Chamada Pública CNPq/ MCTIC/SEMPI No 01/2020 - Empreendimentos e soluções de base tecnológica na área de Grafeno. A FabNS é uma spin-off do LabNS/UFMG (Laboratório de NanoEspectroscopia), sendo constituída majoritariamente por pesquisadores provenientes deste laboratório com experiência no desenvolvimento de equipamentos para a caracterização da nanotecnologia. O grupo vem desenvolvendo expertise na técnica TERS (Espectroscopia Raman melhorada por ponta), por meio da qual um conjunto de materiais e compostos químicos, incluindo nanoestruturas orgânicas e inorgânicas, filmes finos e materiais biológicos pode ser investigado em condições ambientais na escala nanométrica (COSTA; CANÇADO; JORIO, 2021). No contexto da chamada em questão, o desafio estava na 
concepção de um plano de negócio para um serviço de Nanometrologia de Grafenos, tendo como cliente alvo fábricas de grafenos. Neste projeto foram investigados conceitos do serviço em um potencial cliente aqui denominado por FGraf. Ainda, como requisito da chamada, havia a necessidade de se desenvolver um MVP Concierge junto ao potencial cliente.

O estudo se apoiou nos pressupostos e recomendações da Design Science Research (DSR) para desenvolver uma solução gerencial dentro de uma problemática bem definida (VAN AKEN; BERENDS, 2018). A DSR é uma estratégia de pesquisa voltada para criar conhecimento que possa ser utilizado de modo instrumental para projetar e implementar ações, processos e sistemas e atingir resultados esperados na prática. Resultam da DSR proposições de design destinadas a profissionais que vão adotá-las como solução genérica possível para um problema (VAN AKEN; CHANDRASEKARAN; HALMAN, 2016).

Até o ponto de elaboração deste texto o desenvolvimento da proposta contou com 5 meses de trabalho, distribuídos em reuniões semanais entre os autores, 8 encontros pontuais de cerca de 1,5h com representantes da FABNS e FGraf e três workshops de trabalho de cerca de 2,5h envolvendo representantes de todas as equipes.

\section{REVISÃO TEÓRICA}

\subsection{O Desdobramento da Função Qualidade - QFD}

O método QFD é uma forma de comunicar sistematicamente a informação relacionada com a qualidade, traduzindo as reais necessidades de clientes em informações de projeto e explicitando ordenadamente o trabalho relacionado com a obtenção dessa qualidade. $\mathrm{O}$ objetivo central está em alcançar o enfoque da garantia da qualidade durante $o$ desenvolvimento de produtos (bens e serviços) (CHENG; MELO FILHO, 2010a; FREITAS et al., 2015).

O desdobramento da qualidade é realizado utilizando-se fundamentalmente a lógica da causae-efeito, de forma estruturada, hierarquizada e priorizada. O desdobramento parte da voz do cliente (VOC), passando por características da qualidade do produto/serviço final e seus constituintes, até chegar a um determinado valor de um parâmetro de controle de um processo final de produção (CHENG; MELO FILHO, 2010b). Portanto, o processo de desdobramento a partir da VOC passa subsequentemente por todos os fatores necessários para o desenvolvimento do produto e variáveis do negócio como: características de qualidade do produto, funções, características de qualidades dos produtos intermediários e matérias-primas, 
parâmetros de controle, processos, mecanismos, componentes, padrões, entre diversos outros, cujas escolhas e nomenclaturas dependem da natureza de cada projeto (CHENG; MELO FILHO, 2010a).

Os fatores mencionados, assim como a própria VOC (priorizada em ordem de importância) são representados por meio de tabelas que podem ser organizadas em níveis do mais agregado ao mais desdobrado. As relações de causa-e-efeito entre elementos de diferentes tabelas são assinaladas com o apoio de uma matriz de correlações, que não somente é usada para identificar as correlações entre elementos, mas também a intensidade em que tais correlações ocorreriam (figura 1).

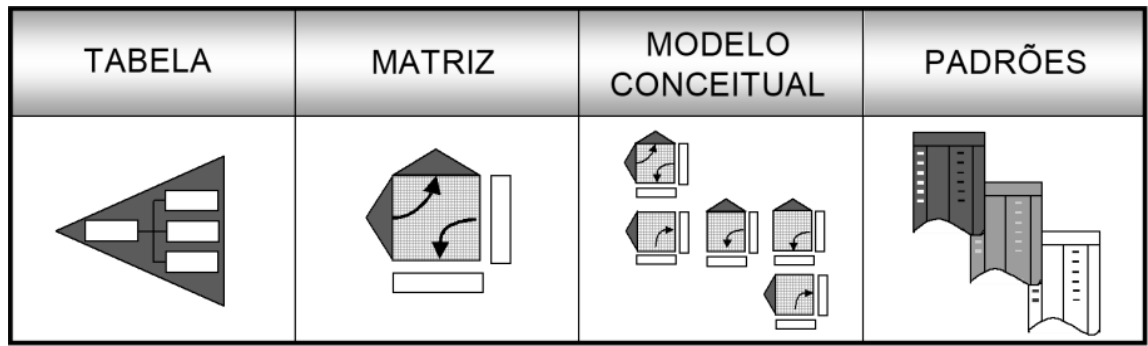

Figura 1 - Unidades operacionais do QFD. Fonte: Cheng e Melo Filho (2010a)

Um Modelo Conceitual é formado pelas tabelas e matrizes de um determinado produto. A decisão sobre a necessidade de efetuar ou não o desdobramento em determinadas dimensões (e.g., tecnologia, custo, confiabilidade) depende dos objetivos de cada trabalho. Por fim, as informações resultantes do desdobramento são transmitidas para as áreas funcionais que realizarão o produto. Um conjunto de padrões é um meio para efetuar essa transmissão (CHENG; MELO FILHO, 2010a).

\subsection{Modelo de referência para o processo de desenvolvimento de startups}

Geralmente, métodos e ferramentas gerenciais para inovação possuem raízes em problemas de grandes empresas de manufatura (SALERNO et al., 2015; BAGNO; SALERNO; SILVA, 2017), ou são excessivamente inspirados em startups associadas a tecnologias de informação (ex. RIES, 2011; BLANK; DORF, 2012). Apesar de rico, esse quadro demanda precauções quanto à aplicação direta dos modelos disponíveis ao contexto de negócios intensivos em ciência. Nesse contexto o modelo P-Start foi selecionado como referência para se delinear as etapas norteadoras do tipo de negócio em questão.

O P-Start foi construído, pesquisado e aplicado em diferentes casos reais a partir da experiência do Núcleo de Tecnologia da Qualidade e Inovação (NTQI/DEP-UFMG) e vem 
sendo continuamente revisado no intuito de equilibrar o viés pragmático e voltado à ação e ciclos rápidos de aprendizado (típico das abordagens contemporâneas de startups digitais) com a disciplina e apoio de ferramentas e métodos estruturados (típico da inovação em empresas estabelecidas) (SOUZA et al., 2017; SOUZA et al., 2018; SOUZA; MELO FILHO; CHENG, 2020). A figura 2 traz uma versão do modelo.

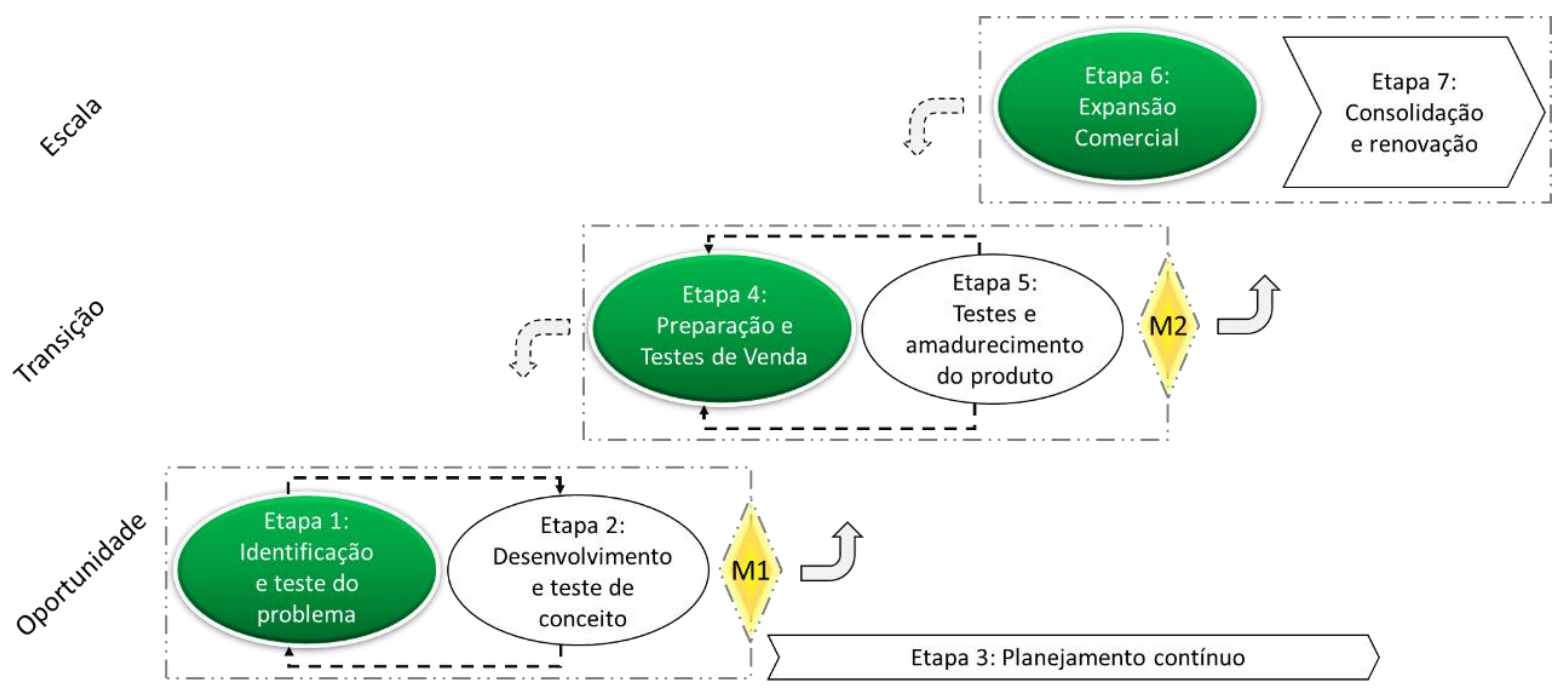

Figura 2 - O P-Start. Fonte: Souza, Melo Filho e Cheng (2020).

\subsection{O Business Model Canvas e o Value Proposition Design}

O Business Model Canvas (BMC) é uma forma representativa sintética das dimensões que definem as linhas gerais de um negócio e é um artefato visual do arcabouço do Business Model Generation (BMG) (OSTERWALDER; PIGNEUR, 2010).

Um modelo de negócio deve descrever a lógica da criação, entrega e captura de valor por parte da empresa. O BMC permite visualizar em página única os blocos constituintes do modelo de negócio, facilitando a averiguação de suas hipóteses (SOUZA et al., 2017). Osterwalder et al. (2014) sugeriram um incremento ao BMC original ao qual chamaram Canvas da Proposta de Valor (Value Proposition Design - VPD) de forma a auxiliar a obtenção do ajuste produto-mercado por meio da utilização de dois artefatos combinados: o perfil do cliente (que auxilia o entendimento dos clientes em termos do que fazem, seus potenciais ganhos e dores) e o mapa de valor (proposta contendo os pontos que visem aliviar "dores" e criar ganhos em atividades importantes para o cliente). 


\subsection{O Blueprint de serviço}

Shostack (1984) cunha o conceito de Blueprint de serviços ao abordar a carência de propostas metodológicas para desenvolvimento e avaliação de serviços. O Blueprint pode ser definido como uma figura ou mapa que representa precisamente o serviço ou um complexo sistema de serviços de forma que diferentes agentes envolvidos em o prover possam compreender e lidar com o serviço de maneira objetiva (VOSS, 2003).

Na sua forma visual, o Blueprint se mostra como um gráfico de progresso do processo da prestação do serviço, desde o contato inicial com o cliente até a conclusão da operação. As etapas são representadas horizontalmente e cada componente (ação relacionada ao serviço) está ordenado verticalmente em planos que representam níveis de proximidade do cliente (SEYRING et al., 2009).

\section{O BUSINESS MODEL - QUALITY FUNCTION DEPLOYMENT (BM-QFD)}

$\mathrm{O}$ acrônimo atribuído à presente abordagem - BM-QFD - deriva das principais referências combinadas no modelo: o BMC e o QFD. Tal combinação já havia sido sugerida por Pitayachaval, Chittrakool e Arjharn (2017) ao identificar semelhanças potenciais entre os campos tipicamente propostos pelo BMC e as possibilidades de desdobramentos abarcáveis pelo QFD, assim como por Souza et al. (2017) que propuseram a aplicação conjunta de métodos no desenvolvimento de startups. O BM-QFD combina cinco métodos de apoio do arcabouço de empreendedorismo tecnológico e inovação para guiar a elaboração dos dois elementos de saída centrais: o Plano de Negócio e o MVP Concierge (figura 3). 


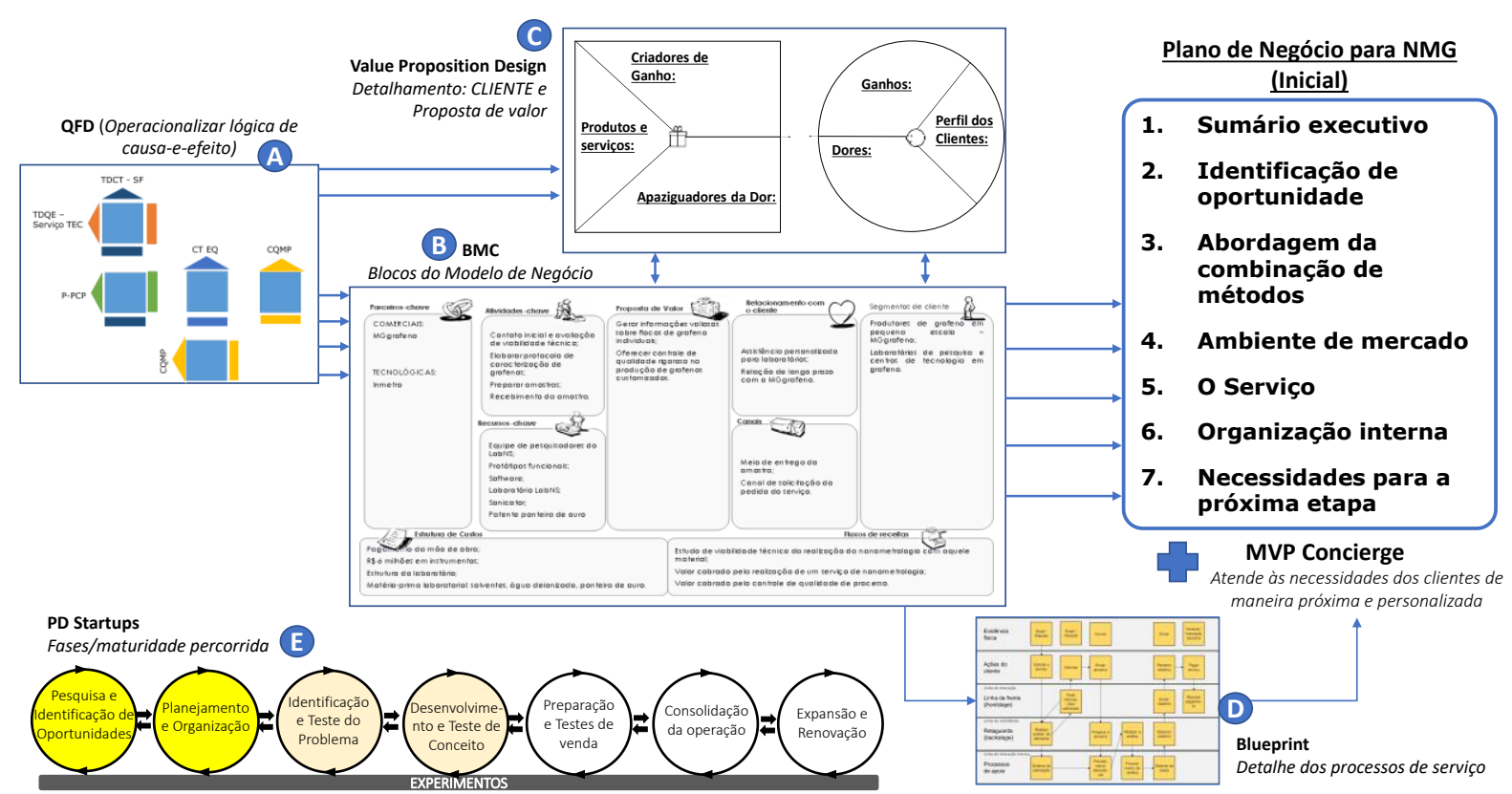

Figura 3 - BM-QFD como combinação de métodos e ferramentas de inovação e empreendedorismo tecnológico. Fonte: dos autores.

O modelo processual (em E na figura 3), foi adaptado a partir do P-Start e consistiu em um framework de sete etapas para auxiliar o desenvolvimento de negócios tecnológicos de origem acadêmica que geralmente se originam pela identificação de oportunidades. Nesta abordagem este processo faz parte do QFD em sua perspectiva mais ampla, representando parte do desdobramento do trabalho. As intensidades de cores em cada etapa do processo como apresentado na figura representam a maturidade do negócio no momento de conclusão da primeira versão do plano de negócios - i.e., maior consolidação e resolução de incertezas associadas às primeiras duas etapas e as duas etapas seguintes em um nível intermediário de avanço. Assim, as necessidades futuras e a definição dos próximos passos (descritas no item 7 do plano de negócios) se norteiam pelo enfoque nas etapas 3 e 4 (da esquerda para direita em E na figura 3) e direcionamento das etapas seguintes do processo.

\subsection{Os outputs do modelo: Plano de Negócios e MVP}

No caso estudado, a equipe da startup e o time de desenvolvimento do plano de negócios avançaram até a entrega da primeira versão do plano, que responde questões e incertezas em coerência com o estágio de maturidade evidenciado nas etapas do processo de desenvolvimento. Contudo, ressalta-se que tal Plano é um documento vivo, objeto de revisões periódicas na medida em que se consolidam etapas anteriores e se avança para as demais. 
Ainda em coerência com o modelo processual, a experimentação contínua foi um elemento transversal entre as etapas e, no contexto do desenvolvimento da proposta aplicada deste estudo, se deu por meio da geração de Produtos Mínimos Viáveis (MVPs). Estes foram delineados não só para responder a perguntas técnicas ou de design do serviço, mas para testar hipóteses fundamentais do negócio da startup como um todo (BRAGA; BAGNO; SOUZA, 2020).

Em suas proposições, Ries (2011) estabelece alguns tipos de MVPs. No contexto específico do estudo aplicado à Nanometrologia de Grafenos, procurou-se delinear o que se chama por MVP Concierge: um MVP concierge atende às necessidades de um cliente específico de forma próxima e personalizada (no caso deste estudo, a FGraf).

Dois níveis distintos de MVP Concierge foram elaborados em sequência:

(i) MVP Concierge em nível abstrato: realizado por meio de workshop QFD com o cliente FGraf, centrado na elaboração, validação e priorização das necessidades identificadas na produção de grafenos. O foco estava na validação das necessidades do cliente, em suas priorizações na qualidade planejada, e nas suas correlações com as funcionalidades e soluções propostas pelo serviço de Nanometrologia, embora realizados num plano mais conceitual. Esta foi uma novidade: o uso do QFD como MVP na aprendizagem validada com potencial cliente.

(ii) MVP Concierge em nível concreto: realização prática de nanocaracterização de grafenos fornecidos pelo cliente, proporcionando a experiência prática da prestação do serviço proposto.

Os níveis "abstrato" e "concreto" de MVP Concierge ora desenvolvidos podem ser compreendidos então como formas distintas de um conceito fatual do serviço. Elas foram úteis não somente para se avaliar e ranquear necessidades do cliente, mas também para ajustar parâmetros do serviço para se obter maiores intenções de adoção da parte deste cliente.

Os resultados do MVP em nível abstrato se refletem nas versões finais das primeiras tabelas do QFD e, consequentemente, no design inicial do negócio e do serviço representado no Business Model Canvas, Value Proposition Design e Blueprint (elementos representativos do negócio e do serviço). Da mesma forma, o MVP em nível concreto foi registrado na forma de relatório técnico evidenciando as capacidades do serviço na prática, com medições e análises 
realizadas sob amostras reais da FGraf, apreciados e validados por ambas as equipes (startup e cliente).

\subsection{Relações de causa-e-efeito como norteador geral do novo negócio e serviço associado}

De forma a servir como método de auxílio à elaboração do Plano de Negócios da empresa nascente, o QFD (representado em A na figura 3) foi utilizado em um nível de análise mais amplo, associado à estratégia e parâmetros macro do negócio - figuras 4 e 5. Dessa forma, partindo-se de uma matriz inicial de correlações entre a voz do cliente e os parâmetros fundamentais do serviço de nanometrologia, o modelo conceitual se desenvolveu por meio da identificação de relações de causa-e-efeito nas dimensões do negócio.

No workshop de QFD, membros convidados do cliente avaliaram, ajustaram e complementaram as qualidades exigidas. Em um segundo momento, já migrando para a Tabela da Qualidade Planejada, os representantes do cliente foram então convidados a estabelecer o grau de importância de cada necessidade identificada à luz da expectativa de contribuição de um serviço de Nanometrologia de Grafenos para sua mitigação ou solução. Neste momento, a cada necessidade foi atribuído um valor numérico (1, 3 ou 5). A partir dessas definições, a Tabela da Qualidade Planejada foi revisada completada em cooperação com a equipe da startup.

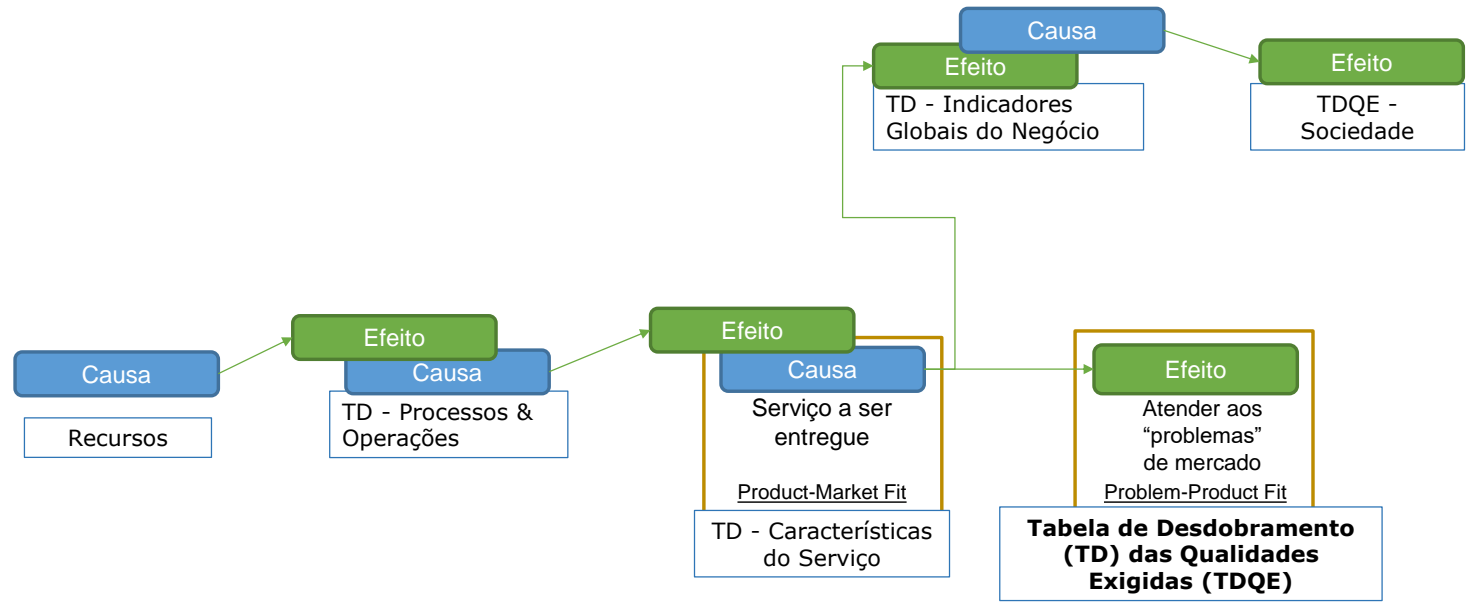

Figura 4 - BM-QFD: Lógica de causa e efeito desdobrada nas dimensões do negócio. Fonte: dos autores. 


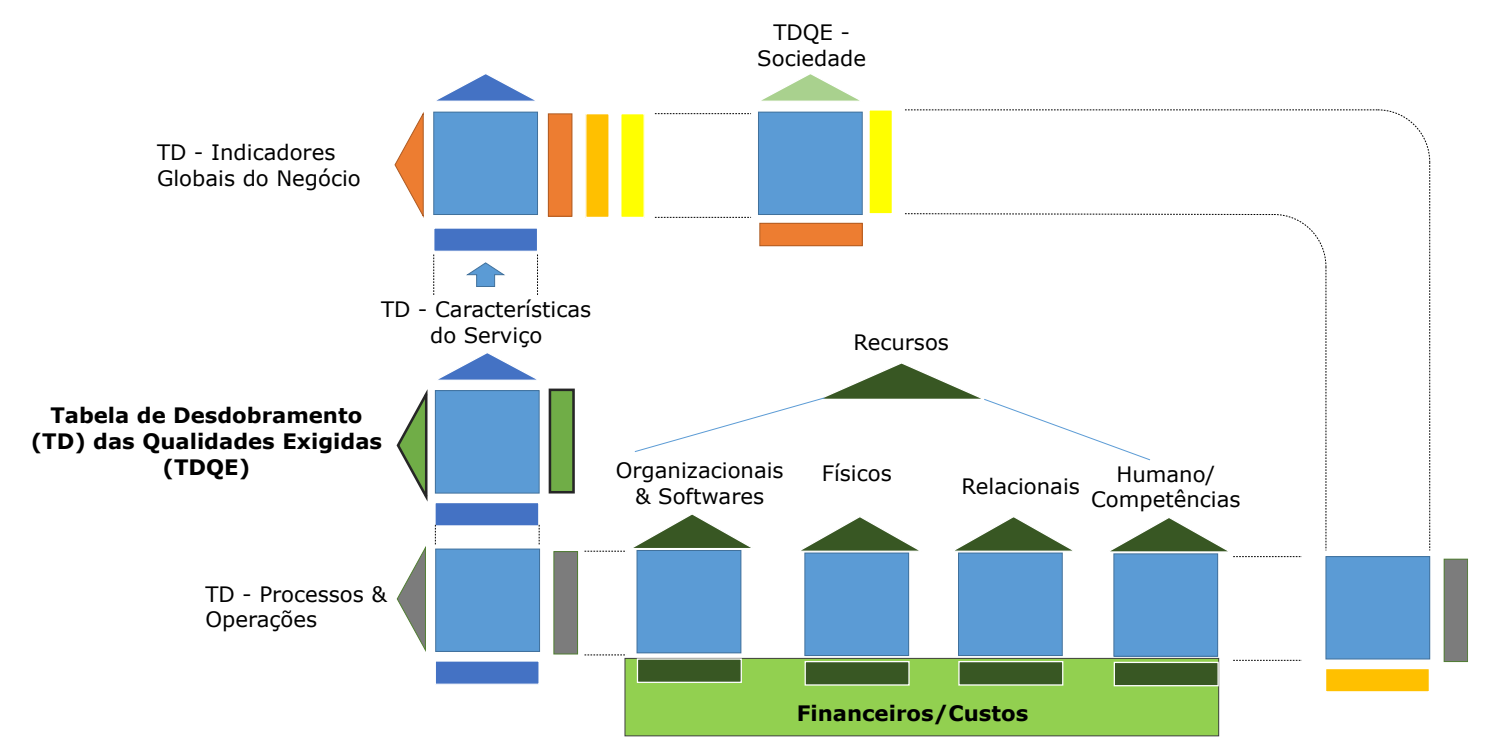

Figura 5 - BM-QFD: Modelo conceitual aplicado ao desenvolvimento de spin-off: caso Nanometrologia de Grafenos. Fonte: dos autores.

$\mathrm{Na}$ sequência deste processo, as características técnicas do serviço de Nanometrologia de Grafenos passam a ser estabelecidas a partir das necessidades do cliente, de modo a caracterizar o primeiro ciclo de relações de causa-e-efeito para o negócio nascente necessidades do cliente satisfeitas como um efeito causado pelas características do serviço prestado. A Tabela de Desdobramento das Características do Serviço foi então organizada segundo cinco dimensões fundamentais do serviço prestado e que guardam maior interação com o cliente: (i) aspectos diretos do sistema de medição com foco nos parâmetros de qualidade da caracterização de grafenos (e.g., resoluções, identificação de defeitos na estrutura nanométrica, quantificação de camadas do material, etc.); (ii) Capacidade de entrega (que traz questões como o tempo de atendimento, flexibilidade do serviço, etc.); (iii) Características do relatório de caracterização (forma, conteúdo...); (iv) Capacidade em P\&D (elementos de respaldo científico e tecnológico do time da startup que legitimam subjetivamente o serviço prestado e atestam a competência embarcada); e, complementarmente, (v) Indicadores do site a ser implementado.

As correlações de cada aspecto do serviço prestado às necessidades do cliente anteriormente identificadas na TQE são então marcadas nos cruzamentos da matriz de acordo com sua intensidade (nenhuma, 1, 3 ou 9). O somatório das correlações identificadas ponderado pelas prioridades demarcadas na Tabela da Qualidade Exigida é finalmente representado na Tabela da Qualidade Projetada que estabelece os percentuais de satisfação do cliente a serem obtidos pela excelência de realização de cada uma das dimensões de qualidade do serviço de especializado em questão. Os demais aspectos internos ao negócio (Processos \& Operações e 
Indicadores Globais do Negócio) podem assim ser desdobrados sucessivamente no modelo conceitual em fases posteriores de maturidade da empresa. A figura 6 mostra o QFD como MVP mais abstrato em workshop para teste, aprendizagem validada, com potencial cliente.

*"Uma lacuna que tem dificultado e crescido a demanda é a realização da análise da qualidade do grafeno (caracterização) puro e especialmente funcionalizado"

Responsável pelo $P \& D$ e funcionalização do grafeno na indústria

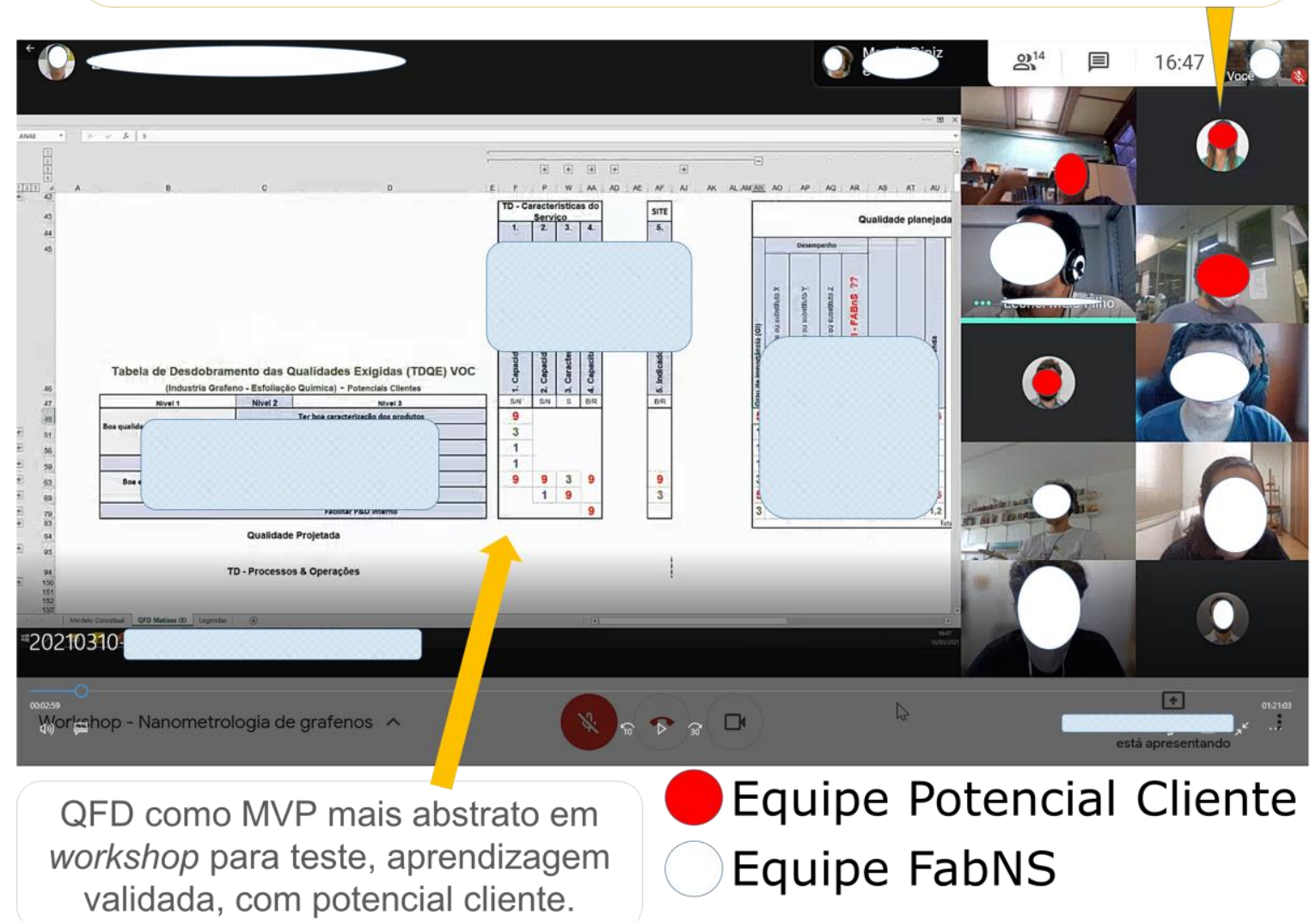

Figura 6 - QFD como MVP mais abstrato em workshop para teste, aprendizagem validada, com potencial cliente. Fonte: dos autores.

\subsection{Artefatos representativos do negócio e do serviço associado}

Como apresentado na figura 3, o Business Model Canvas (BMC) é associado ao Value Proposition Design (VPD) - partes B e C da figura - permitindo o detalhamento e maior amarração entre os campos do BMC de Proposta de Valor e Segmentos de Mercado, que são também os gatilhos principais para o desenho do plano de negócios. Desdobrados em seus parâmetros de avaliação, estes elementos constituem-se das (e realimentam as) informações das tabelas iniciais do QFD (Tabela de Desdobramento da Qualidades exigidas e Tabela de Desdobramento das Características do Serviço). O resultado é uma forma robustecida do 
BMC, apoiada diretamente na validação do cliente e suas prioridades acerca de suas necessidades e percepção do serviço a ser prestado.

Adicionalmente, tendo-se levantado os possíveis processos gerenciais por meio do QFD e percorrido em caráter experimental o fluxo técnico da prestação prática do serviço por meio do MVP concierge concreto, construiu-se um projeto destes processos tangibilizados pelo Blueprint (posicionado em D no modelo genérico da figura 3). Dado que serviços puros são caracteristicamente intangíveis, normalmente não podem ser experimentados com o uso de protótipos em seu sentido convencional (BRAGA; BAGNO; SOUZA, 2020). Nesse sentido, o Blueprint se mostra como ferramenta de boa compreensão dos serviços em complementaridade ao QFD, que atua num nível de análise preliminar e mais interno da definição do serviço.

Juntos, BMC, VPD e Blueprint foram o conjunto de artefatos que sintetizam o negócio e o serviço especializado a ele associado, servindo instrumentos de comunicação sintética e visual (e, por isso, propícios à contínua revisão e reflexão sobre o negócio), sendo todos eles fundamentados nas relações de causa-e-efeito propiciadas pelo QFD em um nível de abstração mais elevado.

\section{CONCLUSÃO}

A presente proposta contou com a combinação e adaptação de métodos consolidados na literatura e usualmente empregados no desenvolvimento de novos produtos e serviços em empresas estabelecidas, e também na atividade empreendedora típica de empresas nascentes. A abordagem resultante, no entanto, é inovadora em relação à combinação e adaptação destes métodos. A motivação para a proposição de uma abordagem metodológica específica encontra-se nas particularidades da presente demanda, a saber, (i) contexto de indústria emergente de alta incerteza, com elos da cadeia de valor por se consolidar e papeis dos agentes em definição; (ii) negócio associado à prestação de serviço de alta tecnologia intensamente baseado em ciência de fronteira, carregando consigo incertezas técnicas e ausência de padrões normativos ou de mercado para se tomar como referência; (iii) necessidade de se articular a demanda e o design do serviço em contato direto com um primeiro cliente potencial arquetípico do mercado vislumbrado.

Como oportunidades para continuidade, espera-se dar sequência ao acompanhamento da startup nas etapas posteriores do negócio, assim como buscar oportunidades de aplicação e 
aprimoramento a partir da experiência de outras startups intensivas em ciência e tecnologia presentes no ecossistema de inovação.

\section{AGRADECIMENTOS}

Os autores agradecem ao CNPq pelo suporte financeiro para realização do trabalho, à equipe FabNS e aos professores Luiz Gustavo de Oliveira Lopes Cançado e Ado Jorio de Vasconcelos pela parceria.

\section{REFERÊNCIAS}

BAGNO, R.B.; SALERNO, M.S.; SILVA, D.O. Models with graphical representation for innovation management: a literature review. R\&D Management, v. 47, n. 4, p. 637-653, 2017.

BLANK, S. Why the lean start-up changes everything. Harvard business review, v. 91, n. 5, p. 63-72, 2013.

BLANK, S.; DORF, B. The startup owner's manual: The step-by-step guide for building a great company. BookBaby, 2012.

BORBA PRÁ, F.; MIGUEL, P.A.C. Evolução na aplicação do QFD: análise de publicações qualificadas em periódicos. Exacta, v. 11, n. 1, p. 89-100, 2013.

BRAGA, I.S.; BAGNO, R.B.; SOUZA, M.L.P.D. Prototipagem: definições, aplicações e oportunidades no empreendedorismo tecnológico. In: BAGNO, R. B.;SOUZA, M. L. P. D., et al (Ed.). Perspectivas sobre o empreendedorismo tecnológico: da ação empreendedora aos programas de apoio e dinâmica do ecossistema. Curitiba: Brazil Publishing, 2020. p.p. 225-265.

CHENG, L.C.; MELO FILHO, L.D.R. QFD: desdobramento da função qualidade na gestão de desenvolvimento de produtos. Editora Blucher, 2010a.

Platform conceptual model in QFD for generic drug. Product: Management and Development, v. 8, n. 1, p. 3-16, 2010 b.

COSTA, M.D.; CANÇADO, L.G.; JORIO, A. Event chronology analysis of the historical development of tipenhanced Raman spectroscopy. Journal of Raman Spectroscopy, v. 52, n. 3, p. 587-599, 2021.

FREITAS, L.S.; MELO FILHO, L.D.R.; CHENG, L.C.; CARMO, M.A.Z. Análise da aplicação do método desdobramento da função qualidade "QFD" em serviços preventivos de polícia. Revista Produção Online, v. 15, n. 1, p. 243-275, 2015.

KALBACH, J. Mapeamento de Experiências: um guia para criar valor por meio de jornadas, blueprints e diagramas. Alta Books, 2019.

LASMAR, T.P.; FREITAS, J.S. O fenômeno das spin-offs acadêmicas. In: BAGNO, R. B.;SOUZA, M. L. P. D., et al (Ed.). Perspectivas sobre o empreendedorismo tecnológico: da ação empreendedora aos programas de apoio e dinâmica do ecossistema. Curitiba: Brazil Publishing, 2020. cap. Cap.18, p.581-600.

OSTERWALDER, A.; PIGNEUR, Y. Business model generation: a handbook for visionaries, game changers, and challengers. John Wiley \& Sons, 2010.

OSTERWALDER, A.; PIGNEUR, Y.; BERNARDA, G.; SMITH, A. Value proposition design: How to create products and services customers want. John Wiley \& Sons, 2014.

PITAYACHAVAL, P.; CHITTRAKOOL, K.; ARJHARN, W. Integration of Business Model Canvas (BMC) and Quality Function Deployment (QFD) to design product. 2017 4th International Conference on Industrial Engineering and Applications (ICIEA), 2017. IEEE. p.76-80.

RATINHO, T.; HARMS, R.; WALSH, S. Structuring the Technology Entrepreneurship publication landscape: Making sense out of chaos. Technological forecasting and social change, v. 100, p. 168-175, 2015. 
RIES, E. The lean startup: How today's entrepreneurs use continuous innovation to create radically successful businesses. Currency, 2011.

SALERNO, M.S.; DE VASCONCELOS GOMES, L.A.; DA SILVA, D.O.; BAGNO, R.B.; FREITAS, S.L.T.U. Innovation processes: Which process for which project? Technovation, v. 35, p. 59-70, 2015.

SEYRING, M.; DORNBERGER, U.; SUVELZA, A.; BYRNES, T. Service blueprinting handbook. Leipzig, Germany: International SEPT Program. University of Leipzig, 2009. 16p.

SHANE, S.A. Academic entrepreneurship: University spinoffs and wealth creation. Edward Elgar Publishing, 2004.

SHOSTACK, G.L. Designing services that deliver. Harvard Business Review, v. 62, 1984.

SOUZA, M.L.; MELO FILHO, L.D.; BAGNO, R.B.; SOUZA, W.C.; CHENG, L.C. A process model integrated to innovation management tools to support technology entrepreneurship. 2018 Portland International Conference on Management of Engineering and Technology (PICMET), 2018. IEEE. p.1-12.

SOUZA, M.L.P.; MELO FILHO, L.D.R.; OLIVEIRA, C.G.G.; ANICETO, M.D.; SILVEIRA, C. Aplicação conjunta de métodos no desenvolvimento de startups: descrição e análise crítica. Anais do Congresso Brasileiro de Inovação e Gestão de Desenvolvimento do Produto (CBGDP), 2017. p.725-734.

SOUZA, M.L.P.D.; MELO FILHO, L.D.R.; CHENG, L.C. P-Start: processo para geração de startups e kit de ferramentas. In: BAGNO, R. B.;SOUZA, M. L. P. D., et al (Ed.). Perspectivas sobre o empreendedorismo tecnológico: da ação empreendedora aos programas de apoio e dinâmica do ecossistema. Curitiba: Brazil Publishing, 2020. cap. Cap. 5, p.111-160.

VAN AKEN, J.; CHANDRASEKARAN, A.; HALMAN, J. Conducting and publishing design science research: Inaugural essay of the design science department of the Journal of Operations Management. Journal of Operations Management, v. 47, p. 1-8, 2016.

VAN AKEN, J.E.; BERENDS, H. Problem solving in organizations. Cambridge university press, 2018.

VOSS, C.A. "Rethinking paradigms of service: service in a virtual environment. International Journal of Operations and Production Management, v. 23, n. 1, p. p.88-104, 2003. 